\title{
Vorbereitung von Instrumententischen Interne Versorgung einer Sprunggelenksfraktur
}

\author{
Regina U. Dries, Anders Mutz
}

\section{Zusammenfassung}

Die Vorbereitung der Instrumententische ist eine der Kernaufgaben der OPPflege. Als einer der wesentlichen Punkte des Prozessablaufs im OP muss der Instrumentenvorbereitung unter Berücksichtigung verschiedener Kriterien, wie z.B. Hygiene, Standardisierung und Einarbeitung von neuen Mitarbeitern und Schülern, große Bedeutung beigemessen werden. Von Seiten des OP-Managements sind die betriebswirtschaftlichen Aspekte und die Qualitätssicherung zu berücksichtigen. Gerade in der Traumatologie und Orthopädie steht ein immer größeres Spektrum an Instrumenten und Implantaten zur Verfügung, welche in effizienter Weise und strukturiert für eine OP vorbereitet werden müssen. Dabei erfordern die zunehmende Technisierung und moderne, zum Teil navigationsunterstützte OP-Verfahren, ein hohes Maß an Lernbereitschaft, Disziplin und Professionalität, um den hohen Qualitätsansprüchen gerecht zu werden. Nur wenn die Vorbereitung der Instrumententische auch Bestandteil des Qualitätsmanagements ist, können unnötige Störfaktoren, wie z.B. Wartezeiten, die Bereitstellung falscher Instrumente oder das Fehlen von Implantaten, vermieden und Überwachungs- und Weiterentwicklungsmaßnahmen im Sinne eines kontinuierlichen Verbesserungsprozesses eingeleitet werden.

\section{Preparation of the Instrument Tables - Closed Management of an Ankle Joint Fracture}

Preparation of the instrument tables is one of the principal tasks operating room management. As one of the major points in the daily use of an operating room, instrument preparation must be taken seriously under consideration of various criteria such as, for example, hygiene, standardisation, training of new personnel and students. From the view point of operating room management, economical and quality control aspects must also be taken into account. Especially in the fields of traumatology and orthopaedics, a wide range of instruments and implants will always be required and must be prepared for an operation in an orderly and structured manner. Here, the increasing use of technical aids and, in part, navigation-supported operating procedures demand a high readiness to learn new techniques, discipline and professionalism in order to ensure the expected high standards of quality. Only when preparation of the instrument tables is a part of quality management cans unnecessary interruptions such as, for example, waiting times, provision of the wrong instruments or the lack of the needed implant is avoided and quality control and further developmental measures be introduced in the sense of a continuous optimisation process.

\section{Einleitung}

Voraussetzung für die korrekte Vorbereitung der Instrumententische ist eine gut funktionierende OP-Logistik.

Komplexe Arbeitsabläufe im Vorfeld einer Operation sind notwendig, um den Ansprüchen des Patienten, des Operateurs und des OP-Personals sowie der Forderung einer betriebswirtschaftlichen Optimierung gerecht zu werden.
Am Beispiel der Sprunggelenksfraktur soll im Folgenden der Prozess der Vorbereitung, von der Zusammenstellung der Siebe bis zur Anordnung der Instrumente auf den Tischen, beschrieben werden.

Das Prinzip der internen Versorgung der Sprunggelenksfraktur mit der Drittelrohrplatte, Kleinfragmentschrauben und Kirschner-Drähten hat sich in den vergangenen Jahren kaum verändert. So hat die Zusammenstellung der Siebe für diese Operation schon beinahe einen historischen Ursprung. Standards, zunächst nicht dokumentiert, wurden von einer Generation der OP-Pflege an die nächste weitergegeben, wobei sich im Laufe der Jahre Modifikationen, entsprechend den Bedürfnissen des ärztlichen Leiters und der Entwicklung von z.B. akkubetriebenen Antriebsmaschinen ergaben. Die Anzahl der zur Verfügung stehenden Siebe und Implantate musste einerseits den gestiegenen OP- Zahlen und andererseits betriebswirtschaftlichen Belangen angepasst werden. Durch die Einführung des Qualitätsmanagements im OP stehen uns heute Methoden und Hilfsmittel zur Verfügung, die es ermöglichen, die Vorbereitung systematisch und für Mitarbeiter transparent zu gestalten.

Zunächst orientieren wir uns an dem OPProgramm. Anhand der Informationen zur Frakturart und der geplanten OP werden benötigte Materialien anhand von „Karteikarten“ auf einem „Containerwagen“ vorbereitet.

Die Zusammenstellung des Materials erfordert keine Fachkenntnisse und kann von nicht examiniertem Personal durchgeführt werden.

\section{Vorbereitung der Sterilcontainer und Einmartikel nach Karteikarten}

Diese Karteikarten (Tab.1-3) stehen sowohl in elektronischer, an jedem EDVArbeitsplatz im OP, als auch in schrift- 
Tab. 1 Vorderseite der Karteikarte „Knöchelfraktur - konventionell“

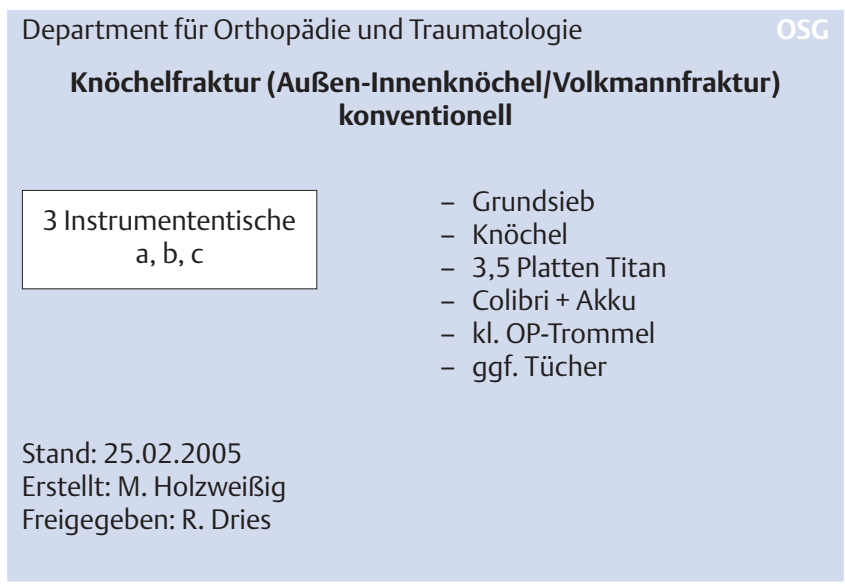

Tab. 2 Vorderseite der Karteikarte „Knöchelfraktur - winkelstabil“

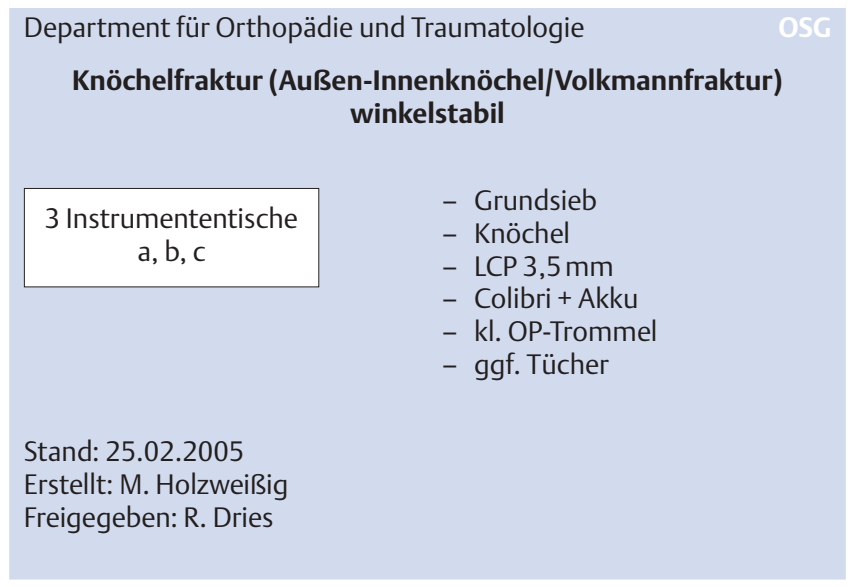

Tab. 3 Rückseite der Karteikarten „Knöchelfraktur“

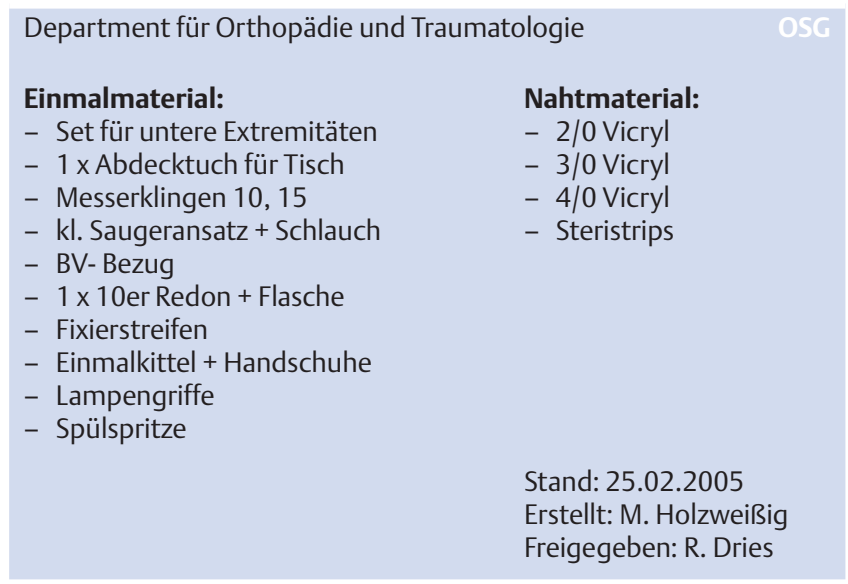

licher Form an zentraler Stelle in den Sterilfluren den Mitarbeitern zur Verfügung.

Die Instrumentensiebe befinden sich ausschließlich in Sterilcontainern, die in Ste- rilfluren in Regalen gelagert werden. Mit Staubdeckeln versehen sind die Standardcontainer ein Jahr haltbar. Die Einmalartikel werden in Modulschrankkörben ebenfalls in den Sterilfluren aufbewahrt.

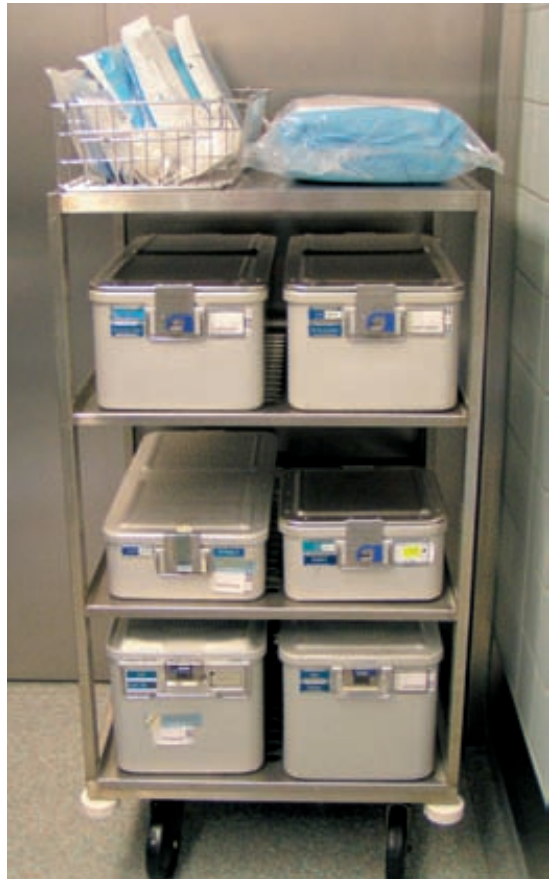

Abb. 1 "Containerwagen“

Die Zusammenstellung des gesamten Materials erfolgt auf so genannten Containerwägen, die dann den entsprechenden Sälen, unabhängig von räumlichen Verschiebungen im OP-Plan, zugeführt werden können (Abb.1).

\section{Inhalt der Instrumentensiebe}

In Anwendervorgaben (Sieblisten in schriftlicher und elektronischer Form) sind die genaue Art und Anzahl der Instrumente, die Verpackungsart, die Packrichtung und Besonderheiten der Funktionsprüfung und Sterilisation dokumentiert. Anhand dieser verbindlichen Vorgabe werden die Siebe von der Zentralen Sterilisationsabteilung aufbereitet. Unter Verwendung dieser Vorgaben werden die Instrumente von dem Springer und dem Instrumentierenden nach einer OP in bereitgestellte Entsorgungscontainer abgelegt und diese mit den Siebnummern den Originalsieben zugeordnet (Abb.2).

\section{Das Grundsieb}

Das Grundsieb kommt bei allen Operationen zur Anwendung und beinhaltet allgemeine und AO-Grundinstrumente. In modifizierter Form steht das Grundsieb auch als „kleines und großes Metallentfernungssieb“ zur Verfügung. In den vergangenen Jahren wurde die Anzahl der Instrumente konsequent reduziert, wodurch eine deutliche Minderung des 


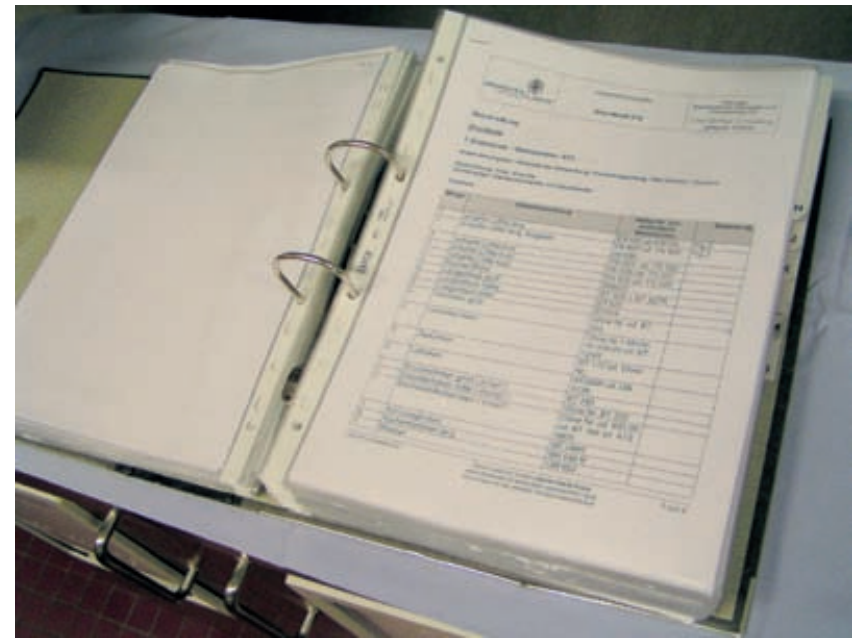

Abb. 2 Ordner mit Anwendervorgaben.

Containergewichtes und eine bessere Übersichtlichkeit im Sieb erreicht wurde (Abb. 3 u. 4).

\section{Das „Knöchel-Sieb“}

In dem Knöchelsieb befinden sich spezielle Osteosynthese-Instrumente wie Repositionszangen in unterschiedlicher Ausführung sowie Kirschner-Drähte und Drahtcerclagen, die für die interne Ver- sorgung der Sprunggelenksfraktur notwendig sein können. Das Knöchelsieb wurde so konzipiert, dass es als Zusatzsieb bei verschiedenen Frakturen und deren Versorgung an den oberen oder unteren Extremitäten verwendet werden kann. So kommt es bei der konventionellen oder winkelstabilen Plattenosteosynthese am Unterarm oder bei der Zuggurtungsosteosynthese (Hohlnadel, 2. Flachzange, Stößel etc.) bei Olekranon- oder
Patellafrakturen gleichermaßen zur Anwendung. Vorteil dieser vielseitigen Anwendbarkeit ist die Reduzierung vorzuhaltender Instrumentensiebe und die bessere Überschaubarkeit, gerade für neue Mitarbeiter. Grundlage für die Bemessung der notwendigen Anzahl der Knöchelsiebe sind die Erfahrungswerte der OP-Leitung, die Infrastruktur hinsichtlich der Aufbereitungszeiten in der Zentralsterilisationen und die statistische Auswertung der OP-Zahlen (Abb.5).

\section{Das Sieb „3,5 Platten Titan“}

Dieses Sieb beinhaltet ein Plattensortiment in Titan für 3,5 und 4,0 mm Schrauben, Titan-Standardschrauben $\varnothing 2,7 / 3,5 /$ $4,0 \mathrm{~mm}$ und das Kleinfragmentinstrumentarium.

Folgende Platten sind enthalten:

- LC-DCP 3.5

- T-Platten 3,5 recht- und schrägwinklig

- Drittelrohrplatten 3,5

- Rekonstruktionsplatten 3,5

- Kalkaneus- und H-Platten 3,5 (Abb.6)

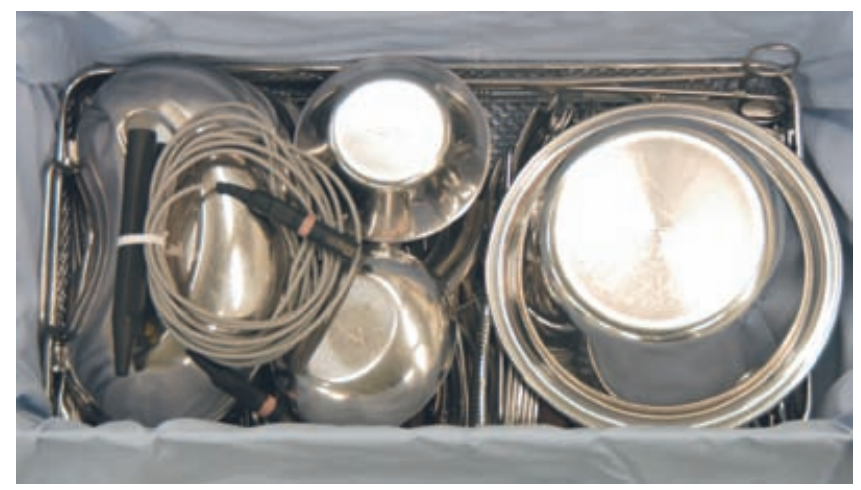

Abb.3 Grundsieb mit Spülschalen.

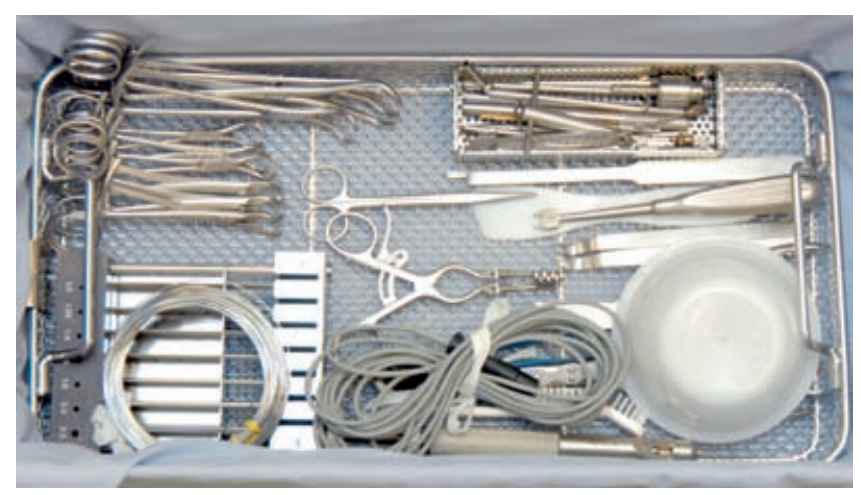

Abb.5 Knöchelsieb

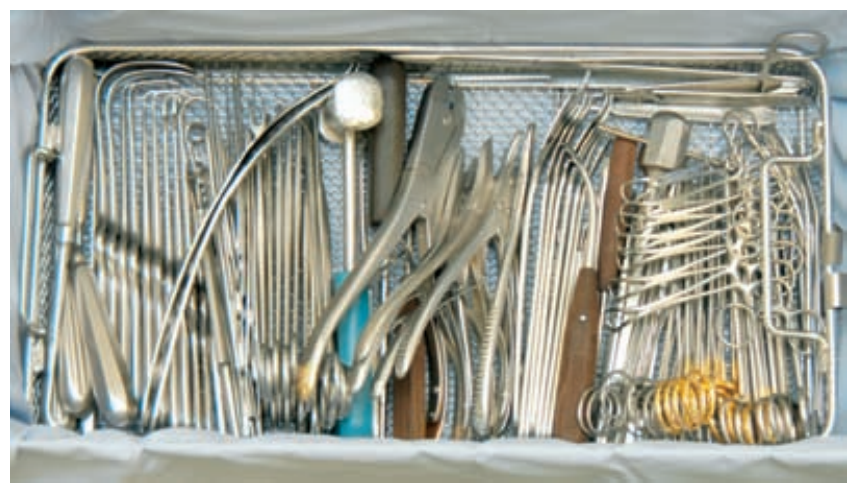

Abb. 4 Grundsieb nach Entnahme der Schalen.

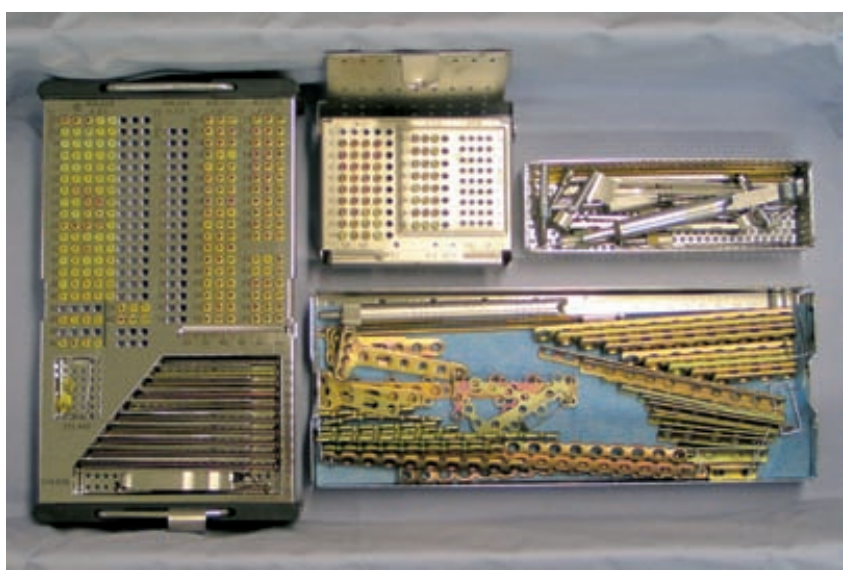

Abb. 6 Sieb 3,5 mm Platten Titan. 


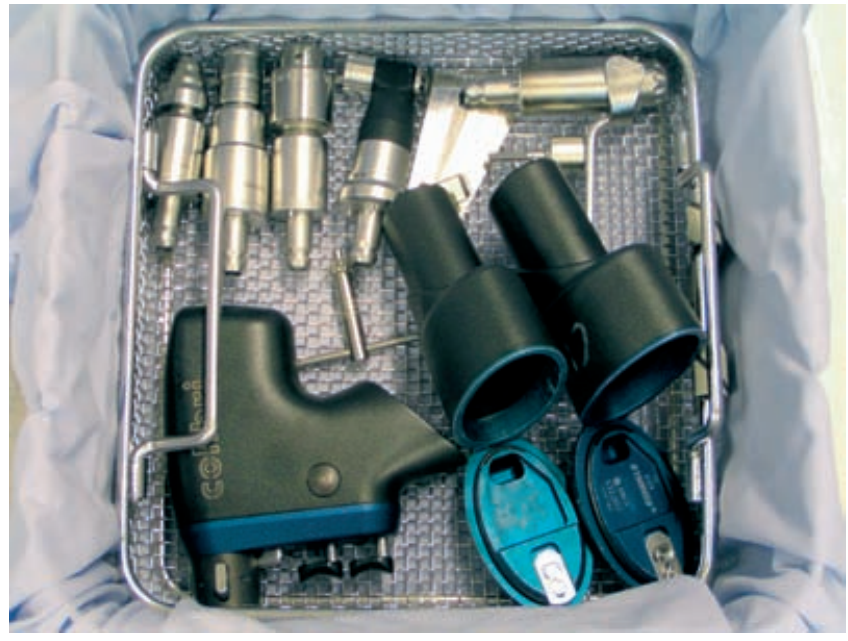

Abb. 7 Colibri

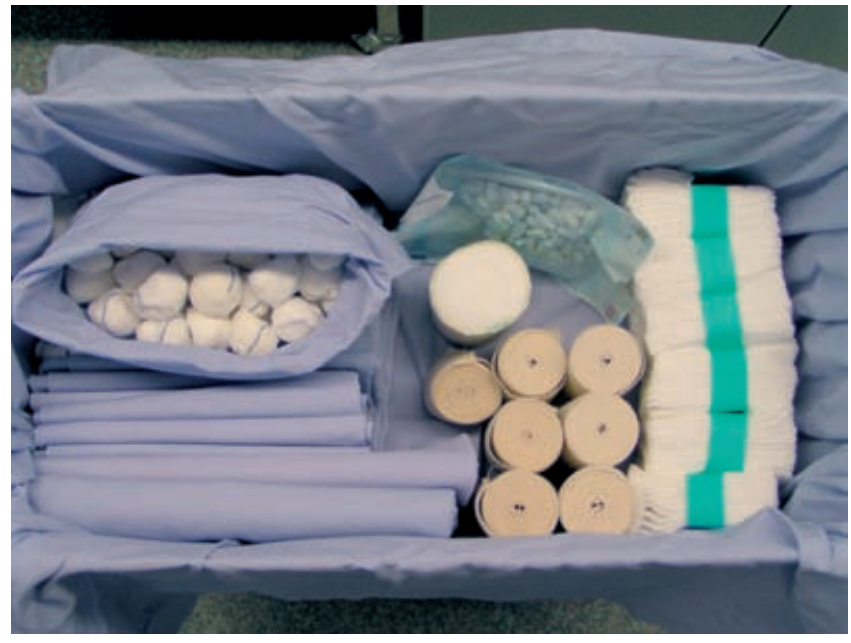

Abb. 8 Kleine OP-Trommel.
In separaten Kleinsetcontainern befinden sich Klavikula-Hakenplatten und Biegeinstrumente für Kleinfragment-Platten.

Zur Anwendung kommt dieses Sieb neben den Sprungelenksfrakturen zum Beispiel auch bei Olekranon-, Unterarm-, Klavikula-, Patella- oder Beckenfrakturen.

\section{Das Sieb „Colibri“}

Der Container „Colibri“ enthält die gleichnamige Synthes Antriebsmaschine mit diversen Aufsätzen und den Akkugehäusen. Die leistungsfähigen Akku-Antriebsmaschinen haben die Druckluftmaschinen bei der Kleinfragment-Osteosynthese ersetzt (Abb.7).

\section{Kleine OP-Trommel}

Inhalt: Verbandstoffe und Tücher. Die große OP-Trommel unterscheidet sich von der kleinen nicht durch die Abmessungen des Containers, sondern durch zusätzlichen Bauchtücher, Streifen und größere elastische Binden (Abb.8).

\section{Tücher}

Der Container „Tücher“ wird nur bei Bedarf geöffnet, wenn die Tücher der „Kleinen OP-Trommel“ nicht ausreichen.

Die Stofftücher werden lediglich zum Schutz vor Kontamination beim Einsatz des C-Bogens über die Patientenabdeckung gelegt oder ggf. zur intraoperativen Unterpolsterung der Extremität.
Anordnung der Instrumententische im OP-Saal

Die Anordnung der Instrumententische im OP-Saal ist definiert und in den OPBeschreibungen des QM-Handbuches dokumentiert:

Beistelltisch
(Sieb- und
Bohrer-
tisch)

A

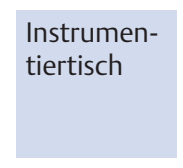

B
Beistelltisch (Sieb- und Verbandtisch)

\section{C}

\section{Verteilung der sterilen Instrumenten-} tisch-Abdecktücher auf den Tischen

$\mathrm{a}=$ Einzelabdecktuch + Verstärkungstuch $\mathrm{b}=$ Tischsack, enthalten im Extremitäten-

Abdeckset

$\mathrm{c}=$ Extremitäten-Abdeckset

In einer dokumentierten Arbeitsanweisung, mit dem Ziel, die korrekte Durchführung sicherzustellen, wird der Abdeckvorgang beschrieben.

\section{Vorbereitung der Instrumententische}

Grundsätzliche Regelungen zur ordnungsgemäßen Instrumentiertätigkeit sind in einer Arbeitsanweisung beschrieben.

Bilder von Instrumententischen, in entsprechenden Ordnern und in elektronischer Form in jedem OP-Saal verfügbar, sollen dazu beitragen, dass die Tische von allen Mitarbeitern systematisch und effizient in immer gleicher Weise vorbereitet werden. Bei dem großen Spektrum an Operationen in unserer Abteilung wird dieses Hilfsmittel nicht nur in der Einarbeitung und Schulung neuer Mitarbeiter und Schüler eingesetzt, sondern wird auch von routinierten Mitarbeitern bei komplexen Vorbereitungen in Anspruch genommen. Die Bilder der Instrumententische ersetzen nicht die theoretische Vorbereitung auf einen Eingriff oder die Kenntnis der Instrumente und des OP-Verlaufes, sondern sind vielmehr als unterstützendes Mittel zu verstehen, um diesen Prozess strukturiert zu gestalten (Abb.9).

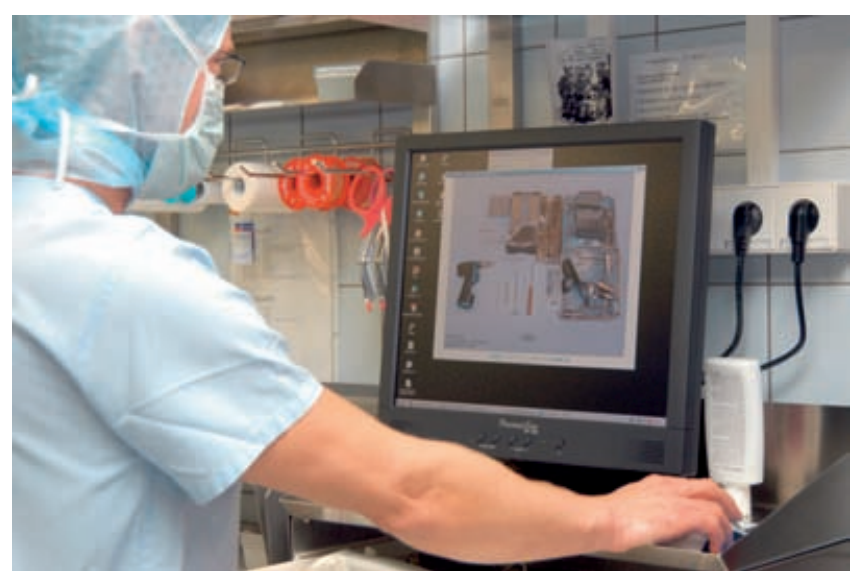

Abb. 9 Bilder der Instrumententische, an jedem PC-Arbeitsplatz verfügbar. 


\section{Anordnung der Instrumente}

Beistelltisch a für die Versorgung einer Weber A-, Boder C-Fraktur

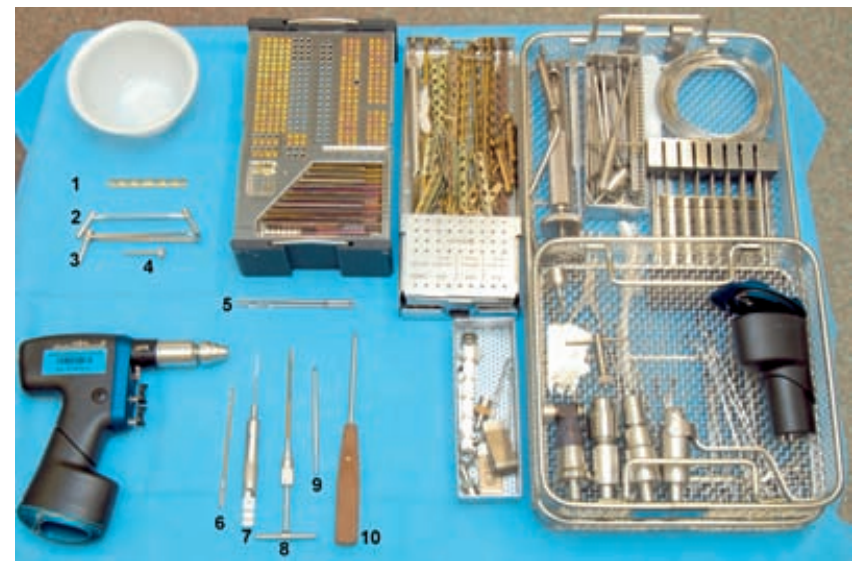

Abb. 10 Tisch a, Beistelltisch (Sieb- und Bohrertisch)

1 = Drittelrohrplatte $3,5 \mathrm{~mm}$

2 = Doppelbohrbüchse 2,5/3,5 mm

3 = Bohrbüchse $3,5 \mathrm{~mm}$

4 = Steckbohrhülse

5 = Bohrer 3,5 mm (Gleitloch für Zugschraube)

6 = Bohrer 2,5 mm (Gewindeloch)

7 = Schraubenmessgerät

8 = Gewindeschneider 3,5 mm

9 = Schraubendreheransatz für die Maschine

10 = Kleinfragment-Schraubendreher

\section{Beistelltisch c}

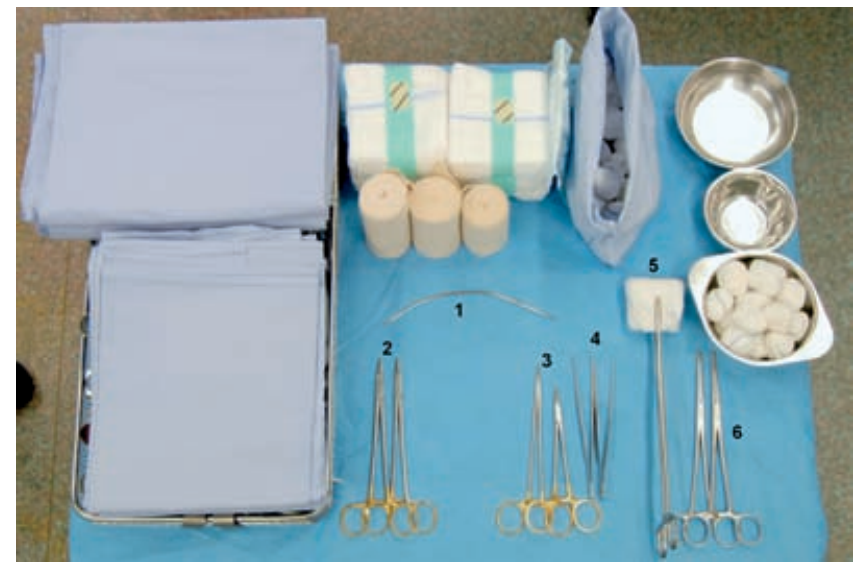

Abb. 12 Beistell-

tisch c

$1=$ Redonspieß 10

$2=$ Nadelhalter

grob (2)

$3=$ Nadelhalter

fein (2)

$4=$ Pinzette chirur-

gisch, fein, mit

Steg

$5=$ Kornzange

(mit Saugtuch)

$6=$ Kocherklemme

(2)

\section{Regina Dries}

OP-Leitung

Anders Mutz

Stellvertr. OP-Leitung

Department für Orthopädie

und Traumatologie

Chirurgische Universitätsklinik Freiburg

Hugstetter Straße 55

79106 Freiburg
$17=$ Kornzange mit Kugeltupfer

$18=$ Kocherklemme

$19=$ Zweizack

$20=$ Elevatorium

21 = Rasparatorium klein, stumpf

22 = Rasparatorium klein, scharf

$23=$ Kocherklemme mittel

$24=$ Overholdt

25 = Einzinker klein, spitz

$26=$ Zweizinker-Wundhaken (2)

$27=$ Lidhaken (2)

$28=$ Wundhaken-Langenbeck ( $2 \times$ klein, $2 \times$ mittel)

$29=$ Knochenhebel - Hohmann ( $2 \times$ spitz, $2 \times$ stumpf)

$30=$ Bipolar-Pinzette und Kabel 